\title{
Scaling Behavior in Thermoelectric Misfit Cobalt Oxides
}

\author{
P. Limelette, ${ }^{1}$ S. Hébert, ${ }^{2}$ V. Hardy, ${ }^{2}$ R. Frésard, ${ }^{2}$ Ch. Simon, ${ }^{2}$ and A. Maignan ${ }^{2}$ \\ ${ }^{1}$ Laboratoire LEMA, UMR 6157 CNRS-CEA, Université F. Rabelais, UFR Sciences, Parc de Grandmont, 37200 Tours, France \\ ${ }^{2}$ Laboratoire CRISMAT, UMR 6508 CNRS-ENSICAEN, 6, Boulevard du Maréchal Juin, 14050 Caen Cedex, France
}

(Received 30 September 2005; published 25 July 2006)

We investigate both thermoelectric and thermodynamic properties of the misfit cobalt oxide $\left[\mathrm{Bi}_{1.7} \mathrm{Co}_{0.3} \mathrm{Ca}_{2} \mathrm{O}_{4}\right]_{0.6}^{\mathrm{RS}} \mathrm{CoO}_{2}$. A large negative magnetothermopower is found to scale with both magnetic field and temperature, revealing a significant spin entropy contribution to thermoelectric properties giving rise to a constant $S_{0} \approx 60 \mu \mathrm{V} \mathrm{K}^{-1}$. Specific heat measurements allow us to determine an enhanced electronic part with $\gamma \approx 50 \mathrm{~mJ}\left(\mathrm{~mol} \mathrm{~K}^{2}\right)^{-1}$ attesting to strong correlations. Thereby, the comparison between cobaltites and other materials reveals a universal behavior of the thermopower slope as a function of $\gamma$, testifying to a purely electronic origin. This potentially generic scaling behavior suggests here that the high room temperature value of the thermopower in misfit cobalt oxides results from the addition of a spin entropy contribution to an enlarged electronic one.

DOI: 10.1103/PhysRevLett.97.046601

PACS numbers: 72.15.Jf, 65.40.-b, 71.27.+a

Since the discovery of large thermopower in metallic $\mathrm{Na}_{x} \mathrm{CoO}_{2}$ and superconductivity in its hydrated form [1,2], the layered cobalt oxides are at the heart of intense current research. Indeed, it was quickly realized that they exhibit striking properties at both low and high temperatures, with a large potential for applications. These properties are resulting from an unusual combination of what is conventionally interpreted as either metallic or insulating features. Even though their resistivity is metalliclike and exhibits rather a low value $[1,3,4]$ as in the so-called bad metal near the Mott metal-insulator transition [5,6], these compounds display a large thermopower (TEP) at room temperature $\left(S_{300 \mathrm{~K}} \approx 138 \mu \mathrm{V} \mathrm{K}^{-1}\right)$, which origin is still debated. Increased thermopower usually stems from either strongly enhanced effective masses through electronic correlations [7-12] or from the entropy of electrons localized in degenerate states [4,13-15]. In most cases, one observes that one effect dominates the other. In contrast, we give below evidence that both scenarios contribute to the enhanced TEP in misfit cobalt oxides.

Similarly to $\mathrm{Na}_{x} \mathrm{CoO}_{2}$, the structure of the incommensurate cobalt oxide $\left[\mathrm{Bi}_{1.7} \mathrm{Co}_{0.3} \mathrm{Ca}_{2} \mathrm{O}_{4}\right]_{0.6}^{\mathrm{RS}} \mathrm{CoO}_{2}$ (abbreviated thereafter $\mathrm{BiCaCoO}$ ) contains single $\left[\mathrm{CoO}_{2}\right]$ layer of $\mathrm{CdI}_{2}$ type stacked with four rocksalt-type layers labeled RS instead of a sodium deficient layer. One of the in-plane sublattice parameters being different from one layer to the other $[4,16]$, the cobaltite $\mathrm{BiCaCoO}$ is a member of a series of compounds having a misfit structure and high thermopower $[3,4,16]$.

We report in this Letter on a detailed experimental study of both thermoelectric and thermodynamic properties of the misfit cobalt oxide $\mathrm{BiCaCoO}$ to determine whether the high room temperature thermopower $S$ could originate from various contributions. In order to identify their signatures, we have performed thermopower measurements as a function of magnetic field and temperature. As reported in Ref. [4], a large negative magnetothermopower is mea- sured at low temperature. Here we show that the latter reveals a scaling behavior with both magnetic field and temperature, suggesting a spin entropy contribution to the thermopower.

Moreover, the determined electronic specific heat coefficient $\gamma \approx 50 \mathrm{~mJ}\left(\mathrm{~mol} \mathrm{~K}^{2}\right)^{-1}$ leads to a universal ratio $S / \gamma T$ independent of interactions that provides a scaling behavior in a wide range of materials including the two misfit cobalt oxides $\mathrm{BiCaCoO}$ and $\left[\mathrm{CoCa}_{2} \mathrm{O}_{3}\right]_{0.6}^{\mathrm{RS}} \mathrm{CoO}_{2}(\mathrm{ab}-$ breviated $\mathrm{CaCoO}$ below). Consequently, the thermopower in this misfit cobalt oxide seems to consist mostly of two contributions: The first one results from a spin entropy part, and the second one originates likely from strongly renormalized quasiparticles.

Thermopower measurements were performed with an experimental setup described in Ref. [17] on a $2 \times 2 \times$ $10 \mathrm{~mm}^{3}$ polycrystalline sintered sample. Figure 1 presents the magnetic field (right inset) and the temperature (left inset) dependences of the thermopower $S$. As appears in the left inset in Fig. 1, three regimes can be distinguished when the temperature $T$ is varied from 300 down to $3 \mathrm{~K}$. Qualitatively often observed in other misfit cobalt oxides $[3,4]$, the thermopower remains first weakly temperature dependent from its room temperature value $S \approx$ $138 \mu \mathrm{V} \mathrm{K}^{-1}$ down to nearly $200 \mathrm{~K}$. Next, $S$ exhibits a quite linear $T$ dependence down to approximately $20 \mathrm{~K}$, followed finally by a strong decrease when the temperature is lowered down to $3 \mathrm{~K}$. In the latter temperature range, namely, below $20 \mathrm{~K}$, the thermopower shown in the right inset in Fig. 1 reveals a strong magnetic field dependence. A large negative magnetothermopower is observed at $3 \mathrm{~K}$ with a reduction of about $65 \%$ between the 0 and $\pm 9 \mathrm{~T}$ values, while a weaker magnetic field effect is seen at higher temperatures, nearly vanishing above $20 \mathrm{~K}$.

The combined influence of both magnetic field and temperature is demonstrated through the complete collapse of the data in Fig. 1 when plotted as a function of $H / T$. 


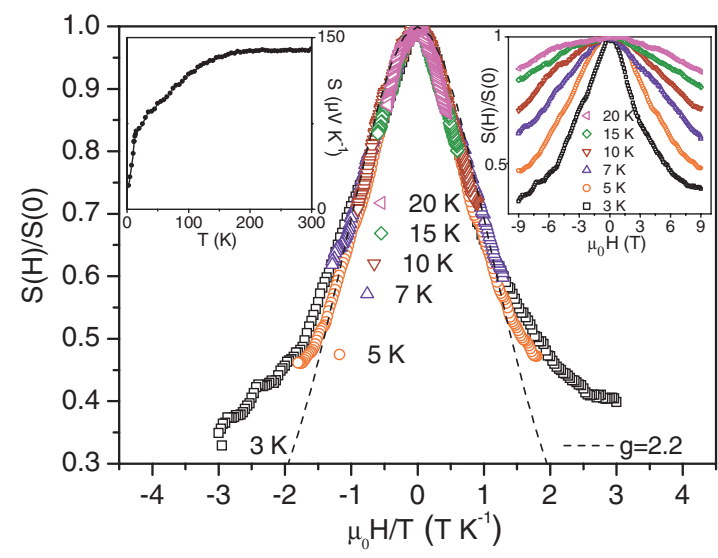

FIG. 1 (color online). Magnetothermopower scaling for a sintered cobalt oxide $\mathrm{BiCaCoO}$. The left and the right insets show, respectively, the temperature and the magnetic field dependences of the hole type thermopower $(S>0)$. The collapse of the data of the right inset as a function of $H / T$ illustrates a spin entropy contribution to the thermopower here normalized to its zero magnetic field value for each temperature as $S(H) / S(H=0)$. The dotted line is the free spins $1 / 2$ entropy following Eq. (1).

Already observed in the layered cobalt oxide $\mathrm{Na}_{x} \mathrm{CoO}_{2}$ [15] (abbreviated $\mathrm{NaCoO}$ below), this scaling behavior is revealed for the first time in a misfit cobaltite, to our knowledge. As mentioned in Ref. [15], such a scaling behavior is a typical signature of a spin entropy reduction involving the freeze of spin fluctuations. Thus, we attribute this magnetothermopower to a collection of spins $1 / 2$ in agreement with the low spin configuration of $\mathrm{Co}^{4+}$ [18], with an entropy $\sigma$ related to the thermopower as $S_{\text {spin }}=$ $\sigma / q, q$ being the electric charge $[13,15]$. Due to this proportionality, the thermopower acquires the same dependence in $H / T$ as the entropy following Eq. (1) that holds for free spins $1 / 2$ [19]

$$
S(x) / S(0)=(\ln [2 \cosh (x)]-x \tanh [x]) / \ln (2) .
$$

Here $x=\left(g \mu_{B} H\right) /\left(2 k_{B} T\right), g$ is the Landé factor, $k_{B}$ the Boltzmann constant, and $\mu_{B}$ the Bohr magneton. It is worth noting that, whereas Eq. (1) accounts qualitatively for the measured magnetothermopower, the quantitative agreement with the experimental results is only efficient with $g=2.2$ for moderate fields, i.e., when $\left|\mu_{0} H / T\right|<$ $1.5 \mathrm{~T} \mathrm{~K}^{-1}$. Nevertheless, we emphasize that the ceramic structure of the studied sample with random orientations of crystallites could lower magnetic field effect without altering scaling properties.

Thermodynamic measurements have been performed on a classical specific heat option of a Quantum Design physical property measurement system on a sample of $34.7 \mathrm{mg}$, in order to determine the electronic contribution to the specific heat. Due to the occurrence of a Schottky anomaly, a direct extrapolation of $C / T$ to zero temperature would overestimate the electronic coefficient $\gamma$, as displayed in Fig. 2.

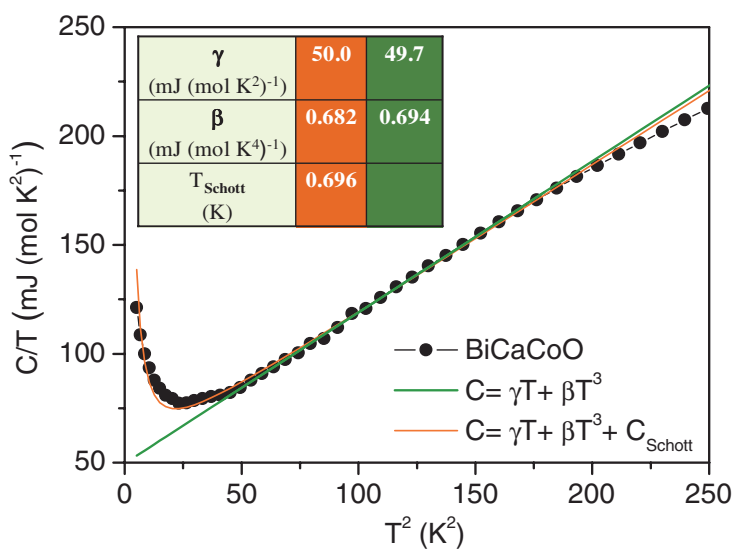

FIG. 2 (color online). Temperature dependence of the specific heat $C$ for a sintered sample as $C / T$ vs $T^{2}$. The linear variation of $C / T$ as a function of $T^{2}$ allows us to determine directly $\gamma \approx$ $50 \mathrm{~mJ}\left(\mathrm{~mol} \mathrm{~K}^{2}\right)^{-1}$. The inset table displays the parameters of the two fitting procedures, namely, with and without the Schottky term.

For $T \gg T_{\text {Schott }}\left(T_{\text {Schott }}\right.$ being the Schottky temperature), the contribution of the Schottky anomaly to the specific heat is given by $C_{\text {Schott }} \sim k_{B}\left(T_{\text {Schott }} / 2 T\right)^{2}$. Introducing this into the fitting procedure yields $T_{\text {Schott }} \approx 0.696 \mathrm{~K}$, and $\gamma \approx 50 \mathrm{~mJ}\left(\mathrm{~mol} \mathrm{~K}^{2}\right)^{-1}$. Since a direct determination of $\gamma$ from the $T^{2}$ behavior of $C / T$ observed over a sizable temperature interval yields the same value within our range of accuracy, we confidently conclude that this rather high value suggests an enhanced electronic effective mass $m^{*}$ which influence upon thermopower is checked below.

As previously mentioned, one can infer that the linear variation of $S$ within the range $20-125 \mathrm{~K}$ in the left inset in Fig. 1 is likely to signal another thermoelectric regime compared to the low temperature regime dominated by a large spin entropy contribution. Theoretically, the electronic thermopower is expected to give rise to a linear temperature dependence contribution with a slope $S / T$ proportional to the effective mass, namely, $\gamma$ [8]. Thus, at a sufficiently low temperature compared to the Fermi energy, the ratio $S / \gamma$ is predicted in this context to be independent of interactions even in a strongly correlated regime as emphasized in Refs. [8,9]. So, in order to check the electronic origin of the linear $T$ dependence of the thermopower, its slope has been plotted in Fig. 3 as a function of $\gamma$ in a wide range of materials $[9,20,21]$ including the cobaltites as $\mathrm{NaCoO}$ [14], $\mathrm{CaCoO}$ [11], $\mathrm{BiCaCoO}$, and even the rhodium misfit oxide $\mathrm{BiBaRhO}$ [22]. In analogy with the prototype compound $\mathrm{NaCoO}$, the electronic specific heat coefficients have been considered for each cobaltite per $\mathrm{CoO}_{2}$, with $\gamma=37 \mathrm{~mJ}\left(\mathrm{~mol} \mathrm{~K}^{2}\right)^{-1}$ for $\mathrm{CaCoO}$.

As a matter of fact, the linear in $T$ behavior of the thermopower is hardly observed in simple metals, where it is hindered by phononic contributions [23]. In contrast, for both misfit cobaltites, it turns out that its prefactor is 


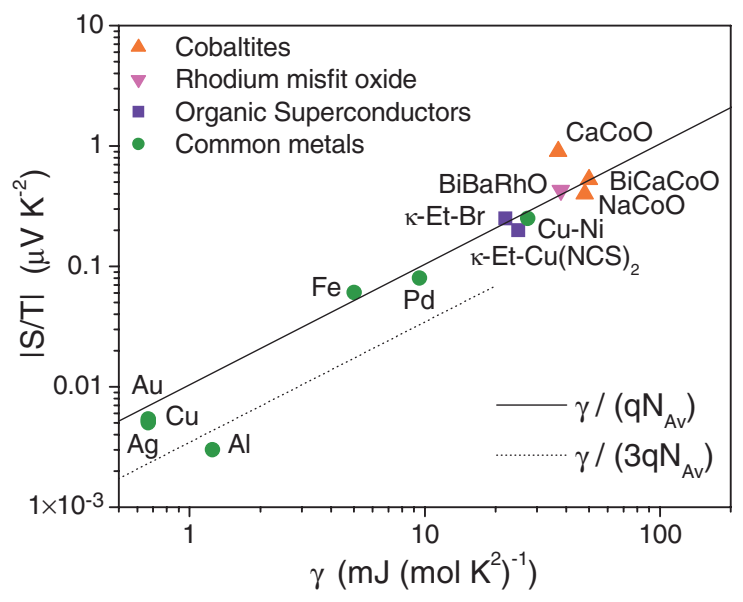

FIG. 3 (color online). Scaling plot of the thermopower slope $|S / T|$ as a function of the electronic specific heat coefficient $\gamma$ for the cobaltites $\mathrm{BiCaCoO}, \mathrm{CaCoO}$ [11], and $\mathrm{NaCoO}$ [14], the rhodium misfit oxide BiBaRhO [22], common metals, and organic superconductors $[9,20,21]$. The straight and dotted lines are discussed in the text.

sufficiently strongly enhanced by the electronic correlations to yield a large temperature domain where the Fermi liquid behavior is observable. Thus, in this temperature range, the $T$ dependence of the thermopower is fully governed by the electronic effective mass and is renormalized by correlations.

In order to give some rather basic theoretical insight into the previous analysis, one may assume following the Landau's Fermi liquid theory [24] that the main effect of the electronic correlations is to renormalize the effective mass. As a consequence, an effective Fermi temperature is defined as $T^{*} \sim T_{F} / m^{*}$ [5], with the bare Fermi temperature $T_{F}$. In this context, the electronic specific heat coefficient $\gamma^{*}$ is

$$
\gamma^{*}=\left(\frac{\pi^{2}}{2}\right) \frac{\delta N_{\mathrm{Av}} k_{B}}{T^{*}},
$$

with the doping $\delta$ and the Avogadro number $N_{\mathrm{Av}}$ [21]. Obviously, one recovers in Eq. (2) a proportionality between $\gamma^{*}$ and the effective mass through $1 / T^{*}$ as expected. The electronic thermopower $S^{*}$ can be inferred in the framework of this basic approach for $T \ll T^{*}$ as:

$$
S^{*}=\left(\frac{\pi^{2}}{6}\right) \frac{k_{B} T}{q T^{*}} .
$$

Since $S^{*}$ in Eq. (3) is also proportional to the effective mass, it results that the ratio $S^{*} / \gamma^{*} T$ in Eq. (4) is thereby independent of interactions as already noted in the context of the dynamical mean field theory of strongly correlated systems $[5,7,8]$

$$
\frac{S^{*}}{\gamma^{*} T}=\left(\frac{1}{3 \delta q N_{\mathrm{Av}}}\right)
$$

Besides, it follows from Eq. (4) that most of the coefficients in Eq. (2) and (3) cancel out in the ratio $S^{*} / \gamma^{*} T$ that is only doping dependent. The latter dependence can thus explain the differences observed in Fig. 3 between experimental points and the straight line plotted following Eq. (4) with $\delta=1 / 3$. In particular, the plotted dotted line with $\delta=1$ accounts quite well for aluminum that is characterized by a quasispherical Fermi surface and one electron per atom. So this analysis suggests that the two misfit cobalt oxides $\mathrm{CaCoO}$ and $\mathrm{BiCaCoO}$ have a hole doping $(S>0)$ lying between nearly 0.13 and 0.33 .

Because the relation (3) contains no free parameter besides $T^{*}$, its direct quantitative comparison with the experimental results should fully determine the effective Fermi temperature. In order to compare the thermopower kinetic component in both misfit cobaltites, one needs to substract the asymptotic value of the spin entropy contribution $S_{0}$ to make the linear $T$ dependence vanishing with temperature.

So, one observes in Fig. 4 as a function of the reduced temperature $T / T^{*}$ a collapse of the different curves with the determined effective Fermi temperatures $T_{\mathrm{BiCaCoO}}^{*} \approx$ $255 \mathrm{~K}$ and $T_{\mathrm{CaCoO}}^{*} \approx 140 \mathrm{~K}$. Moreover, Fig. 4 clearly displays that the breakdown of the linear dependence of the thermopower seems to occur for the two compounds at the same reduced temperature of the order of $T^{*} / 2$, indicating thus a possible crossover fully controlled by correlations. Finally, we emphasize that, while the thermopower is nearly constant above $T^{*}$, its asymptotic value differs from one cobaltite to the other one. We stress here that the high temperature regime $T>T^{*}$ involves likely incoherent electronic excitation, namely, of higher energy and is thus out of the scope of this low energy analysis. It is

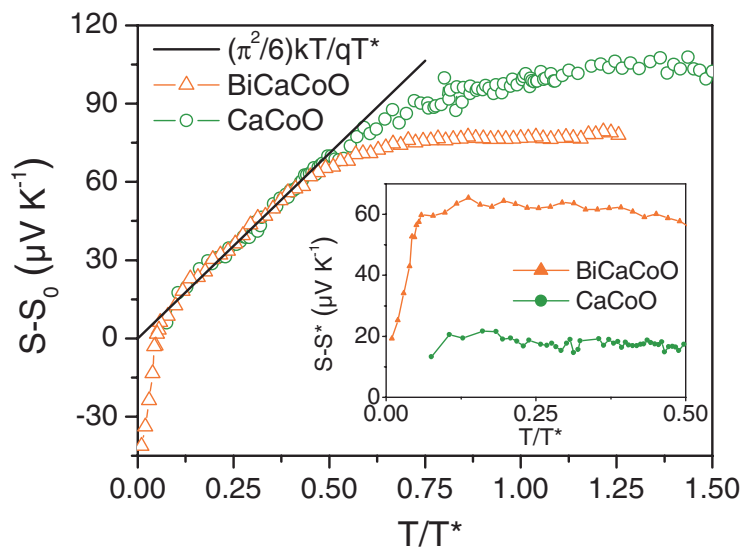

FIG. 4 (color online). Thermopowers $\left(S-S_{0}\right)$ as a function of the reduced temperature $T / T^{*}$, with $S_{0}$ the asymptotic value of the spin entropy contribution and $T^{*}$ the effective Fermi temperature. A comparison is made between the two cobalt oxides $\mathrm{BiCaCoO}\left(S_{0} \approx 60 \mu \mathrm{VK} \mathrm{K}^{-1}, T^{*} \approx 255 \mathrm{~K}\right)$ and $\mathrm{CaCoO}\left(S_{0} \approx\right.$ $\left.20 \mu \mathrm{VK}^{-1}, T^{*} \approx 140 \mathrm{~K}\right)$, while the straight line shows $S^{*}$ following Eq. (3). The inset displays the spin entropy contribution $S_{\text {spin }}=S-S^{*}$ for the two cobaltites. 
worth noting that the temperature dependence of the spin entropy contribution $S_{\text {spin }}$ can also be extracted from the experimental thermopower subtracting the kinetic contribution defined with the corresponding $T^{*}$, as $S_{\text {spin }}=(S-$ $\left.S^{*}\right)$. Therefore, the spin entropy contribution in the inset in Fig. 4 yields the constant $S_{0} \approx 20 \mu \mathrm{V} \mathrm{K}^{-1}$ for $\mathrm{CaCoO}$ and leads asymptotically to $S_{0} \approx 60 \mu \mathrm{V} \mathrm{K}^{-1}$ for $\mathrm{BiCaCoO}$. One must emphasize that the latter value agrees with the theoretical value $S_{0}=k_{B} \ln (2) / q \approx 60 \mu \mathrm{V} \mathrm{K}^{-1}$ expected for one free spin $1 / 2$ per Co site in the $\mathrm{CoO}_{2}$ layers.

Let us mention that a similar coexistence between delocalized electrons and localized spins has already been evidenced in the context of the heavy electrons materials within the two fluid Kondo lattice model [25]. While the underlying physics of the latter results from distinct well identified energy scales (Kondo temperature, intersite coupling, crystal field effect splitting), here this coexistence may involve different competing effects. First, it is worth noting that the triangular arrangement of the cobalt sites in this quasi-two-dimensional structure makes the system naturally predisposed to magnetic frustration. As emphasized in Ref. [10], such a system with a strong Coulomb repulsion could be also characterized by charge frustration leading to a metallic state with a tendency for short-range charge ordering. In addition to this frustration effect, the misfit structure between the $\left[\mathrm{CoO}_{2}\right]$ and the RS layers may introduce disorder by distorting some of the $\mathrm{CoO}_{6}$ octahedrons and lowering the local intersite coupling that could also localize electrons. So for now, in order to investigate both frustration and disorder effects, we believe that further x-ray and neutron scattering experiments are highly desirable.

To conclude, we have performed both thermopower and thermodynamic measurements in the misfit cobalt oxide $\mathrm{BiCaCoO}$ focusing on the identification of various regimes. Magnetothermopower experiments have demonstrated through a scaling behavior a spin entropy contribution, giving rise to a constant $S_{0} \approx k_{B} \ln (2) / q$ above nearly $20 \mathrm{~K}$. While specific heat measurements yield $\gamma \approx$ $50 \mathrm{~mJ}\left(\mathrm{~mol} \mathrm{~K}^{2}\right)^{-1}$, the thermopower $T$-linear dependence $|S / T|$ is found to scale with $\gamma$ in a wide range of materials including the cobaltites $\mathrm{BiCaCoO}, \mathrm{CaCoO}$, and $\mathrm{NaCoO}$. Therefore, we conclude that the thermopower in these misfit cobalt oxides behaves as if it were composed by two components, a kinetic one originating from quasiparticles renormalized by electronic correlations and a spin entropy contribution. Finally, we would like to address that the ratio $S / \gamma T$ seems to provide an efficient probe to check the influence of electronic correlations upon thermopower and should be put to experimental testing in other misfit cobaltites as well as in other strongly correlated systems.

We are grateful to D. Jérome, K. Behnia, G. Kotliar, and A. Georges for useful discussions.

[1] I. Terasaki, Y. Sasago, and K. Uchinokura, Phys. Rev. B 56, R12685 (1997).

[2] K. Takada et al., Nature (London) 422, 53 (2003).

[3] A. C. Masset et al., Phys. Rev. B 62, 166 (2000).

[4] A. Maignan et al., J. Phys. Condens. Matter 15, 2711 (2003).

[5] A. Georges, G. Kotliar, W. Krauth, and M. J. Rozenberg, Rev. Mod. Phys. 68, 13 (1996).

[6] P. Limelette et al., Phys. Rev. Lett. 91, 016401 (2003).

[7] G. Pálsson and G. Kotliar, Phys. Rev. Lett. 80, 4775 (1998).

[8] J. Merino and R.H. McKenzie, Phys. Rev. B 61, 7996 (2000).

[9] K. Behnia, D. Jaccard, and J. Flouquet, J. Phys. Condens. Matter 16, 5187 (2004).

[10] O. I. Motrunich and P. A. Lee, Phys. Rev. B 69, 214516 (2004).

[11] P. Limelette et al., Phys. Rev. B 71, 233108 (2005).

[12] R. Frésard et al., Phys. Lett. A 303, 223 (2002).

[13] W. Koshibae, K. Tsutsui, and S. Maekawa, Phys. Rev. B 62, 6869 (2000).

[14] K. Koumoto, I. Terasaki, and M. Murayama, Oxide Thermoelectrics (Research Signpost, India, 2002).

[15] Y. Wang, N. Rogado, R. Cava, and N. Ong, Nature (London) 423, 425 (2003).

[16] H. Leligny et al., C.R. Acad. Sci. Ser. Gen., Ser. 2 2, 409 (1999).

[17] J. Hejtmanek et al., Phys. Rev. B 54, 11947 (1996).

[18] T. Mizokawa et al., Phys. Rev. B 71, 193107 (2005).

[19] B. Diu, C. Guthmann, D. Lederer, and B. Roulet, Physique Statistique (Hermann, Paris, 1989).

[20] F. J. Blatt and R. H. Kropschot, Phys. Rev. 118, 480 (1960); R. J. Gripshover, J. B. VanZytvelt, and J. Bass, ibid. 163, 598 (1967); I. Weinberg, ibid. 157, 564 (1967); S. Soffer, J. A. Dreesen, and E. M. Pugh, ibid. 140, A668 (1965).

[21] N.W. Ashcroft and N.D. Mermin, Solid State Physics (Holt-Saunders, Philadelphia, 1976).

[22] Y. Klein, S. Hébert, D. Pelloquin, V. Hardy, and A. Maignan (to be published).

[23] J. Weiss and D. Lazarus, Phys. Rev. B 10, 456 (1974).

[24] A. A. Abrikosov, L. P. Gorkov, and I. E. Dzyaloshinski, Methods of Quantum Field Theory in Statistical Physics (Dover, New York, 1975).

[25] S. Nakatsuji, D. Pines, and Z. Fisk, Phys. Rev. Lett. 92, 016401 (2004). 